\title{
Effect of a magnetic field on the thermodynamic uncertainty relation
}

\author{
Hyun-Myung Chun, ${ }^{1}$ Lukas P. Fischer, ${ }^{1}$ and Udo Seifert ${ }^{1}$ \\ ${ }^{1}$ II. Institut für Theoretische Physik, Universität Stuttgart, 70550 Stuttgart, Germany
}

(Dated: April 2, 2019)

\begin{abstract}
The thermodynamic uncertainty relation provides a universal lower bound on the product of entropy production and the fluctuations of any current. While proven for Markov dynamics on a discrete set of states and for overdamped Langevin dynamics, its status for underdamped dynamics is still open. We consider a two-dimensional harmonically confined charged particle in a magnetic field under the action of an external torque. We show analytically that, depending on the sign of the magnetic field, the thermodynamic uncertainty relation does not hold for the currents associated with work and heat. A strong magnetic field can effectively localize the particle with concomitant bounded fluctuations and low dissipation. Numerical results for a three-dimensional variant and for further currents suggest that the existence of such a bound depends crucially on the specific current.
\end{abstract}

\section{INTRODUCTION}

Nonequilibrium systems are characterized by constantly flowing currents and inevitably accompanied dissipation. Some currents, such as the particle current in a molecular motor or the energy current in a heat engine, can be used for practical purposes. In order to optimize the practical use of the currents, it is necessary to suppress two factors: uncertainty and dissipation. Uncertainty of currents due to thermal fluctuation makes a system less predictable and dissipation should be minimal to sustain the efficient operation of the system.

Recent studies have revealed that a universal trade-off between uncertainty and dissipation exists in nonequilibrium systems [1] 3 . This trade-off, called the thermodynamic uncertainty relation, states that suppressing uncertainty costs more dissipation, and conversely, reducing dissipation causes larger uncertainty. Since its first discovery [1], the thermodynamic uncertainty relation has been proven for Markov jump dynamics on a discrete set of states [2, 4, 5] and Langevin dynamics [5, 9] for continuous variables. Generalizations to finite time statistics [10 12] and to periodically driven systems [4, 13, 14] have also been reported.

The standard proofs of the thermodynamic uncertainty relation, however, assume that the observables referring to the state of the system are even under time reversal. The proven validity of the thermodynamic uncertainty relation is thus limited to the overdamped regime where inertia is negligible. The thermodynamic uncertainty relation for systems with variables that are odd under time reversal, like in underdamped Langevin dynamics, remains a puzzling problem. Two examples have shown intriguing features. First, for a driven underdamped particle in a periodic potential, no violation of the thermodynamic uncertainty relation has been observed for the particle current [15. Second, for ballistic transport in multiterminal conductors, a violation is observed only in the presence of a magnetic field [16, 17]. For near-equilibrium systems with broken Onsager symmetry, which can be caused by magnetic fields, a mathematical explanation for the violation was provided in Ref. [17. In addition, a weaker trade-off for underdamped Langevin dynamics, in which dissipation is replaced by a quantity called dynamical activity [15, 18, has been reported.

In this paper, we investigate a physical mechanism by which a magnetic field acting on an underdamped particle causes a violation of the thermodynamic uncertainty relation. To this end, we consider a charged Brownian particle driven by an external torque in the presence of a magnetic field [19. We focus on the work done by the external torque on the particle which can be written in form of an integrated current. We show that the Lorentz force induced by a strong magnetic field in underdamped dynamics localizes the motion of the particle into a smaller area, which in this case leads to a decrease of dissipation. On the other hand, the uncertainty of the work current does not increase with a strong magnetic field, due to a fast decrease of current fluctuations. Thus a strong magnetic field can decrease the dissipation without increasing the uncertainty, which results in a violation of the thermodynamic uncertainty relation.

\section{MODEL}

We first consider two-dimensional motion of a charged particle in a fluid in equilibrium at temperature $T$. The position and the velocity of the particle are denoted by column vectors $\boldsymbol{x}=\left(x_{1}, x_{2}\right)^{\mathrm{T}}$ and $\boldsymbol{v}=\left(v_{1}, v_{2}\right)^{\mathrm{T}}$ where the superscript ' $\mathrm{T}$ ' stands for the transpose. The particle is trapped by a conservative force associated with a harmonic potential $V(\boldsymbol{x})=k \boldsymbol{x}^{2} / 2$ with a spring constant $k$. A constant magnetic field $\boldsymbol{B}$ of strength $B=|\boldsymbol{B}|$, whose direction is perpendicular to the plane of the particle motion, induces a magnetic Lorentz force $\boldsymbol{g}(\boldsymbol{v})=q B\left(v_{2},-v_{1}\right)^{\mathrm{T}}$ with the charge of the particle $q$. In addition to these forces, an external torque $\boldsymbol{f}(\boldsymbol{x})=$ $\kappa\left(x_{2},-x_{1}\right)^{\mathrm{T}}$ now drives the particle out of equilibrium, resulting in a circular particle current. The overdamped analogon of this system without a magnetic field has been thoroughly studied [20 22 and experimentally realized [23, 24].

Overall, the motion of the particle is governed by the 
underdamped Langevin equation

$$
\begin{aligned}
\dot{\boldsymbol{x}}(t) & =\boldsymbol{v}(t), \\
m \dot{\boldsymbol{v}}(t) & =-k \boldsymbol{x}(t)+\boldsymbol{f}(\boldsymbol{x}(t))-\gamma \boldsymbol{v}(t)+\boldsymbol{g}(\boldsymbol{v}(t))+\boldsymbol{\xi}(t),
\end{aligned}
$$

where $m$ is the mass of the particle and $\gamma$ is a damping coefficient. The Gaussian white noise $\boldsymbol{\xi}(t)=\left(\xi_{1}(t), \xi_{2}(t)\right)^{\mathrm{T}}$ is characterized by $\left\langle\xi_{i}(t)\right\rangle=0$ and $\left\langle\xi_{i}(t) \xi_{j}\left(t^{\prime}\right)\right\rangle=$ $2 \gamma k_{\mathrm{B}} T \delta_{i j} \delta\left(t-t^{\prime}\right)$ with the Boltzmann constant $k_{\mathrm{B}}$. The angle brackets $\langle\cdot\rangle$ stand for the ensemble average. Hereafter, we set the charge of the particle $q$ and the Boltzmann constant $k_{\mathrm{B}}$ to unity. For brevity, we introduce collective notations $\boldsymbol{z}=(\boldsymbol{x}, \boldsymbol{v})^{\mathrm{T}}$ and $\boldsymbol{\eta}=(\mathbf{0}, \boldsymbol{\xi})^{\mathrm{T}}$ so that the equations of motion are concisely written as

$$
\dot{\boldsymbol{z}}(t)=-\mathrm{A} \boldsymbol{z}(t)+\boldsymbol{\eta}(t)
$$

with

$$
\mathrm{A}=\frac{1}{m}\left(\begin{array}{cccc}
0 & 0 & -m & 0 \\
0 & 0 & 0 & -m \\
k & -\kappa & \gamma & -B \\
\kappa & k & B & \gamma
\end{array}\right)
$$

The Gaussian white noise $\boldsymbol{\eta}(t)$ is characterized by $\left\langle\eta_{i}(t)\right\rangle=0$ and $\left\langle\eta_{i}(t) \eta_{j}\left(t^{\prime}\right)\right\rangle=2 D_{i j} \delta\left(t-t^{\prime}\right)$ where $D_{i j}$ are the elements of the matrix $\mathrm{D}=\left(\gamma T / \mathrm{m}^{2}\right) \operatorname{diag}\{0,0,1,1\}$. This linear Langevin equation is a multivariate OrnsteinUhlenbeck process [25].

When the real parts of all eigenvalues of the matrix A are positive, the system is stable and the probability distribution of $\boldsymbol{z}$ eventually converges to a steady state. This stability condition of the system is given by (see Appendix A for details)

$$
\gamma k+\kappa B-\kappa^{2} m / \gamma>0 .
$$

Consequently, the particle is confined by the potential if the strength of torque $|\kappa|$ is moderate enough to satisfy the stability condition, otherwise the particle escapes from the potential.

The external torque does work on the particle with rate

$$
\dot{W}(t)=\boldsymbol{f}(t) \cdot \boldsymbol{v}(t)=\kappa\left(x_{2}(t) v_{1}(t)-x_{1}(t) v_{2}(t)\right)
$$

This work either increases the energy of the particle or is dissipated into the surrounding as heat with rate $\dot{Q}(t)=(\gamma \boldsymbol{v}(t)-\boldsymbol{\xi}(t)) \circ \boldsymbol{v}(t)$ [26, 27] where the symbol $\circ$ denotes the Stratonovich product. With the total energy of the particle $E=m \boldsymbol{v}^{2} / 2+k \boldsymbol{x}^{2} / 2$, these definitions are consistent with the first law of thermodynamics $\dot{E}(t)=\dot{W}(t)-\dot{Q}(t)$.

The mean dissipation in a nonequilibrium system is quantified by the entropy production rate $\sigma$. The total entropy production consists of the change in the stochastic entropy of the system and the medium entropy production which is given by $S_{\text {med }}=Q(t) / T$ in our case [27]. In a steady state, both the stochastic entropy and the total energy of the system do on average not change over
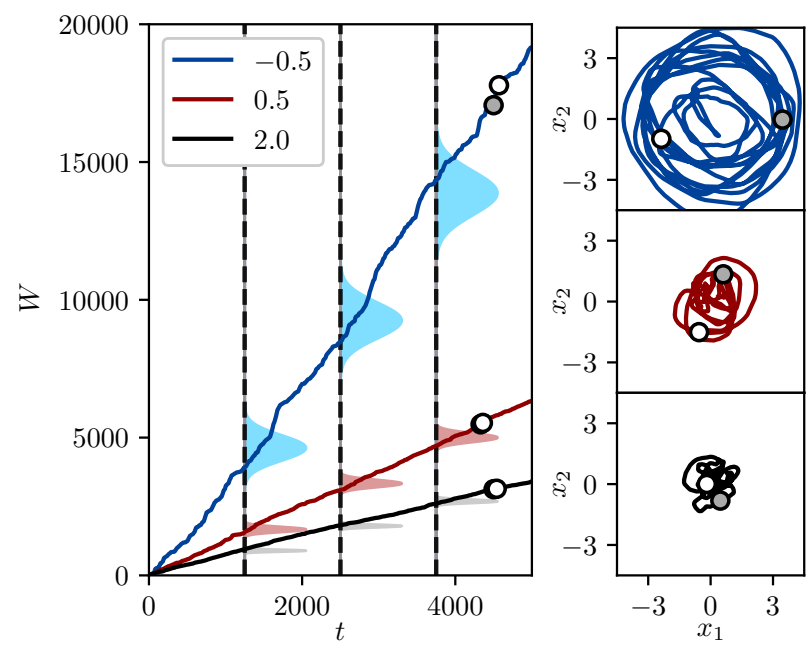

FIG. 1. The left panel shows three sample trajectories of the work done by an external torque on a charged Brownian particle for different strengths of the magnetic field $B$. The strengths of the magnetic field are $-0.5,0.5$, and 2.0 from top to bottom in both panels. The distributions behind the time series represent the distributions of the work done up to the given time. The other parameters are fixed as $m=1$, $\gamma=1, k=2, \kappa=1$, and $T=1$. The right panel shows typical trajectories of the particle corresponding to the three different strengths of the magnetic field. The gray (white) circle denotes the start (end) point of each trajectory.

time. Thus the mean entropy production rate is given by $\sigma=\left\langle\dot{S}_{\text {med }}\right\rangle_{\mathrm{ss}}=\langle\dot{Q}\rangle_{\mathrm{ss}} / T=\langle\dot{W}\rangle_{\mathrm{ss}} / T$. The subscript of $\langle\cdot\rangle_{\mathrm{ss}}$ indicates that the ensemble average is taken in a steady state which we will also take as initial distribution.

In the following section, we analyze the behavior of the rate of work as a current in order to investigate the effect of a magnetic field on the thermodynamic uncertainty relation. Since the work current is proportional to the angular momentum of the particle, it can be interpreted as a measure of the circular particle current. Also, the work current is equivalent to the heat current as the mean value and diffusion coefficient of the heat current coincides with those of the work current in the steady state.

\section{EFFECT OF A MAGNETIC FIELD ON THE THERMODYNAMIC UNCERTAINTY RELATION}

The integrated work current $W(t)=\int_{0}^{t} d t^{\prime} \dot{W}\left(t^{\prime}\right)$ depends on the trajectory of the particle and thus is a stochastic variable. As a consequence of the torque, the work $W(t)$ increases on average as also visible in Fig. 1 . The deviation of the work from its mean value is quantified through the diffusion coefficient

$$
D_{W}=\lim _{t \rightarrow \infty} \frac{1}{2 t}\left(\left\langle W(t)^{2}\right\rangle_{\mathrm{ss}}-\langle W(t)\rangle_{\mathrm{ss}}^{2}\right) .
$$


The uncertainty of the fluctuating work current is characterized by the squared relative uncertainty

$$
\epsilon_{W}^{2}=2 D_{W} /\langle\dot{W}\rangle_{\mathrm{ss}}^{2} .
$$

It measures how fast the variance increases with respect to the mean growth rate of the integrated current. The thermodynamic uncertainty relation states that the quantity $\mathcal{Q}_{W}=\epsilon_{W}^{2} \sigma / 2$, which we shall refer to as the uncertainty product, is bounded from below by the Boltzmann constant which is set to unity here.

In polar coordinates, the work current is given by $\dot{W}=$ $-\kappa r^{2} \dot{\theta}$ with the radial distance $r=|\boldsymbol{x}|$ and the polar angle $\theta=\tan ^{-1}\left(x_{2} / x_{1}\right)$. The steady state probability distribution of the Ornstein-Uhlenbeck process can be obtained by standard procedures [19, 21, 25]. For a given $r$, the conditional distribution of the angular velocity $\dot{\theta}$ is a Gaussian with mean value $-\kappa / \gamma$ and variance $T /\left(m r^{2}\right)$. Thus the mean work current is proportional to the mean squared radial distance, which is given by

$$
\langle\dot{W}\rangle_{\mathrm{ss}}=\frac{\kappa^{2}}{\gamma}\left\langle r^{2}\right\rangle_{\mathrm{ss}}=\frac{2 \kappa^{2} T}{\gamma k+\kappa B-\kappa^{2} m / \gamma}
$$

as derived in detail in Appendix A. The stability condition (4) guarantees that (8) is always positive. Since the mean values of the work and heat current are balanced in the steady state, the mean entropy production rate scales as $\sigma=\langle\dot{W}\rangle_{\mathrm{ss}} / T \sim B^{-1}$ for large $B$ provided that $\kappa B>0$.

This decrease of dissipation with increasing strength of the magnetic field is a result of a stronger localized motion of the particle. In the absence of a magnetic field, the sign of $\kappa$ determines the rotational direction of the motion. A positive $\kappa$ leads to a clockwise rotation around the center of potential. By supplying energy to the particle, the external torque only increases the radial distance. Once the magnetic field is turned on, the induced Lorentz force pushes the particle either inward or outward of the potential depending on the sign of $\kappa B$ as shown in the right panels of Fig. 1. When $\kappa B<0$, the magnetic field reinforces the tendency to increase the radial distance. When $\kappa B>0$, however, the Lorentz force makes the particle prefer to head towards the center of the potential. Thus, as $B$ increases, the particle becomes localized in a smaller area around the center. This also reduces the dissipation since the work rate, Eq. (8), and thus also the heat rate, decrease with the particle being localized in a smaller area. The localization effect only occurs in the presence of both the external torque and the magnetic field.

On the other hand, the squared relative uncertainty does not increase with stronger $B$. The expressions for the mean current and the diffusion coefficient show that the relative uncertainty even decreases with stronger $B$ in the small mass limit because the diffusion coefficient decreases much faster than the mean current. Since the Langevin equation is linear, the exact expressions for the mean current and the diffusion coefficient can be obtained by expanding the scaled cumulant generating function [22, 28, 29] as shown in detail in Appendix B. In terms of the dimensionless parameters $\kappa_{0}=\kappa / k$, $B_{0}=B / \gamma$ and $m_{0}=k m / \gamma^{2}$, they turn out to be given by

$$
\langle\dot{W}\rangle_{\mathrm{ss}}=\frac{2 \kappa_{0}^{2}}{1+\kappa_{0} B_{0}-\kappa_{0}^{2} m_{0}}\left(\frac{T}{\tau}\right),
$$

and

$$
D_{W}=\frac{2 \kappa_{0}^{2}\left[1+\kappa_{0}^{2}\left(1+3 m_{0}\right)+\kappa_{0}^{3} m_{0} B_{0}\right]}{\left(1+\kappa_{0} B_{0}-\kappa_{0}^{2} m_{0}\right)^{3}}\left(\frac{T^{2}}{\tau}\right),
$$

where $\tau=\gamma / k$ is a parameter with the dimension of time. While the leading term in $D_{W}$ for large magnetic fields is $\left(2 \kappa_{0}^{2} m_{0} T^{2}\right) /\left(\tau B_{0}^{2}\right)$, the mean work rate only scales with $1 / B_{0}$. As a consequence the relative uncertainty $\epsilon_{W}^{2}$, Eq. 7), converges to a constant as $B_{0}$ increases. In the small mass limit $m_{0} \rightarrow 0$ the leading order of $D_{W}$ even becomes $B_{0}^{-3}$ which leads to a decreasing relative uncertainty in this limit. An alternative derivation of the scaling order of $D_{W}$ is presented in Appendix C. Overall, the converging or decreasing relative uncertainty $\epsilon_{W}^{2}$ for large magnetic fields cannot balance the decrease of the mean entropy production $\sigma$. As a consequence the thermodynamic uncertainty relation can be broken in the presence of a strong magnetic field.

Using the expressions for the mean current and the diffusion coefficient, Eqs. (9) and (10), the uncertainty product can be explicitly written as

$$
\mathcal{Q}_{W}=\frac{1+\kappa_{0}^{2}\left(1+3 m_{0}\right)+\kappa_{0}^{3} m_{0} B_{0}}{\left(1+\kappa_{0} B_{0}-\kappa_{0}^{2} m_{0}\right)^{2}} .
$$

Without the magnetic field $\left(B_{0}=0\right)$, the thermodynamic uncertainty relation $\mathcal{Q}_{W} \geq 1$ is restored. For given $\kappa_{0}$ and $B_{0}$, the uncertainty product is monotonously decreasing with decreasing $m_{0}$. Thus the uncertainty product is greater than

$$
\lim _{m_{0} \rightarrow 0} \mathcal{Q}_{W}=\frac{1+\kappa_{0}^{2}}{\left(1+\kappa_{0} B_{0}\right)^{2}},
$$

which is greater than $1+\kappa_{0}^{2}$ for $\kappa_{0} B_{0} \leq 0$. Consequently, the underdamped model with $\kappa_{0} B_{0} \leq 0$ obeys the usual bound on $\mathcal{Q}_{W}$ as shown in Fig. 2, For $\kappa_{0} B_{0}>0$, the small inertia bound in 12 is minimal for $\kappa_{0}=B_{0}$ and then simply reads

$$
\mathcal{Q}_{W} \geq \frac{1}{1+B_{0}^{2}},
$$

where equality holds when $\kappa_{0}=B_{0}=0$. Following the optimizations done so far the bound (13) becomes saturated when $\kappa_{0}=B_{0}$ and $m_{0} \rightarrow 0$. As sketched in the positive half of Fig. 2, the bound goes below 1 and approaches zero for large $B_{0}$. In this regime the thermodynamic uncertainty relation does not hold and the uncertainty product can even approach zero. We have 


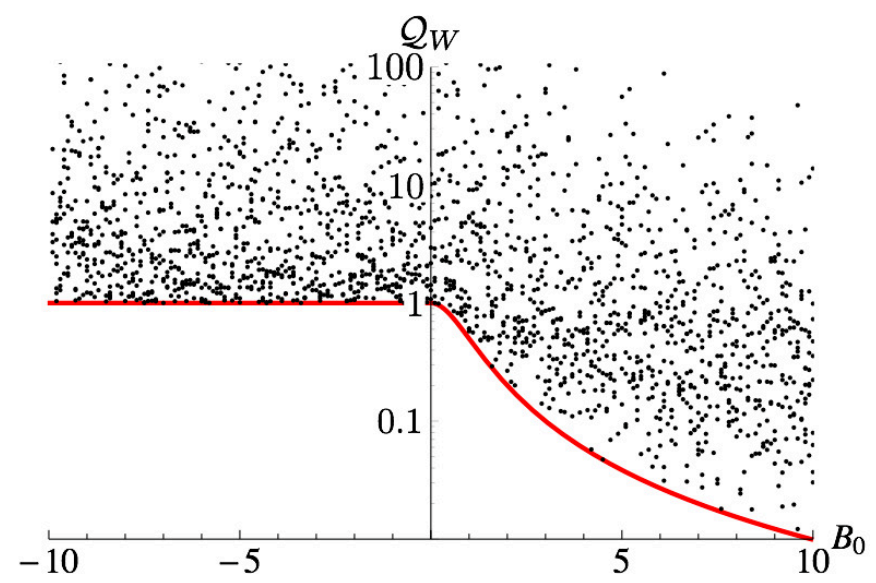

FIG. 2. The scatter plot shows that the uncertainty product is always greater than the bound depicted as solid red line. The bound takes on 1 for $\kappa_{0} B_{0}<0$ and otherwise corresponds to Eq. 13. Each data point is determined by a parameter set $\left(\kappa_{0}, m_{0}, B_{0}\right)$ that satisfies the stability condition. The parameters $\kappa_{0} \in[0,10]$ and $m_{0} \in[0,1]$ are randomly chosen, while $B_{0} \in[-10,10]$ is chosen at a constant interval of 0.1 .

thus shown that the localization of the motion of the particle due to the magnetic field, which occurs only when $\kappa_{0} B_{0}>0$, is responsible for the violation of thermodynamic uncertainty relation in 13 .

We briefly discuss further inequalities that have been derived in recent works. A bound obtained from the fluctuation theorem for entropy production for general dynamics [30] can be applied to our model only when there is no magnetic field and hence the fluctuation theorem holds. On the other hand, another inequality termed hysteretic thermodynamic uncertainty relation 31, which takes into account the time-reversed dynamics, can be applied to our model for a weak enough magnetic field. For strong magnetic fields, however, the time-reversed dynamics can become unstable as a consequence of the asymmetry with respect to $B$ in the stability condition in (4). In their respective range of validity both inequalities provide exponentially weaker lower bounds on the uncertainty product than 13 does.

\section{UNCERTAINTY PRODUCT FOR OTHER CURRENTS}

So far we have focused on the work current. In general one can consider arbitrary currents. The lower bound on the uncertainty product then can depend on the current of interest. For example, for ballistic transport in multiterminal conductors, the uncertainty product of the particle current is bounded from below by a non-zero constant [16], whereas that of a generalized current defined in the linear response theory can come arbitrarily close to 0 by optimizing the chemical potentials applied to the conductor [17. Based on numerical results we show that
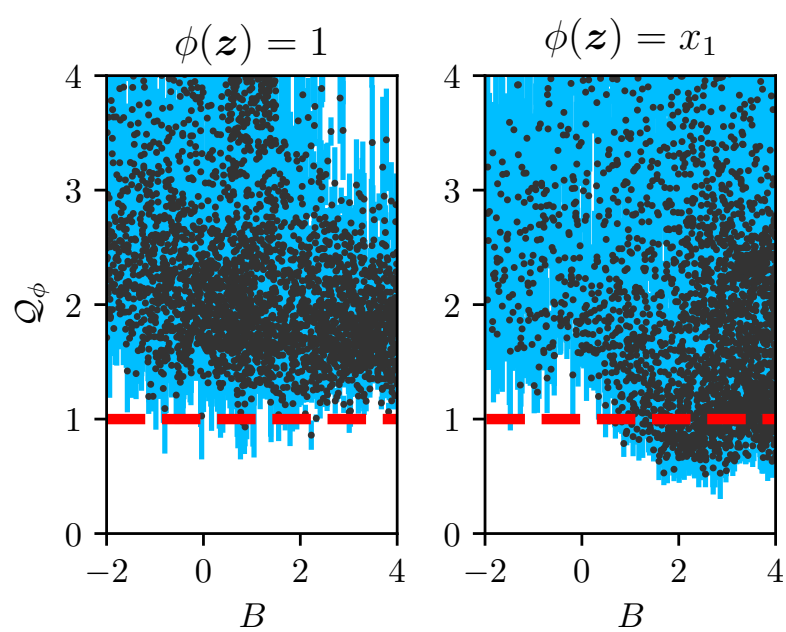

FIG. 3. Numerical results for the uncertainty product $\mathcal{Q}_{\phi}$ for the winding number current 14 with two different weighting factors $\phi(\boldsymbol{z})=1$ (left) and $\phi(\boldsymbol{z})=x_{1}$ (right). Each dot represents one stable parameter set with $B \in[-2,4], \kappa \in$ $[0.05,5], k \in[0,4], \gamma \in[0.1,10], T \in[0.5,1.5]$. The mass $m$ is set to unity. The vertical blue lines are an estimator for the error. The error is estimated by calculating the $25 \%$ percentile for the slope of the mean current and its variance on an ensemble of trajectories. The entropy production rate $\sigma$ is calculated on base of the analytical results Eq. (8). The error of the uncertainty product then follows by propagation of error from the error of the mean current and the error of the diffusion coefficient.

a similar effect can be observed for the present model as well.

In the case of the charged Brownian particle, the work current $\dot{W}=-\kappa r^{2} \dot{\theta}$ can be interpreted as an angular current $\dot{\theta}$ with a weighting factor $-\kappa r^{2}$. An alternative way of quantifying the angular current is to look at the more general class of winding number currents that count the number of crossings of the positive $x_{1}$-axis with a weighting factor $\phi(\boldsymbol{z})$. Formally this current is given by

$$
\begin{aligned}
j_{\phi}(t)= & \frac{1}{t} \int_{0}^{t} d s \phi(\boldsymbol{z}(s)) \chi_{x_{1}\left(s^{-}\right)} \chi_{x_{1}\left(s^{+}\right)} \\
& \times\left[\chi_{x_{2}\left(s^{-}\right)}\left(1-\chi_{x_{2}\left(s^{+}\right)}\right)-\left(1-\chi_{x_{2}\left(s^{-}\right)}\right) \chi_{x_{2}\left(s^{+}\right)}\right]
\end{aligned}
$$

where $\chi_{x}$ is an indicator that is 1 if $x>0$ and 0 otherwise. The times $s^{-}$and $s^{+}$denote the time right before and after $s$, respectively. The advantage of the winding number current is that it could be directly measured in experimental situations without tracing complete trajectories of the particle. The uncertainty product associated with the current $j_{\phi}$ is given as

$$
\mathcal{Q}_{\phi}=\frac{D_{\phi}}{\left\langle j_{\phi}\right\rangle_{\mathrm{ss}}^{2}} \sigma
$$

where $D_{\phi}$ is the diffusion coefficient of the time integrated current $J_{\phi}(t)=\int_{0}^{t} d t^{\prime} j_{\phi}\left(t^{\prime}\right)$. We numerically calculate 


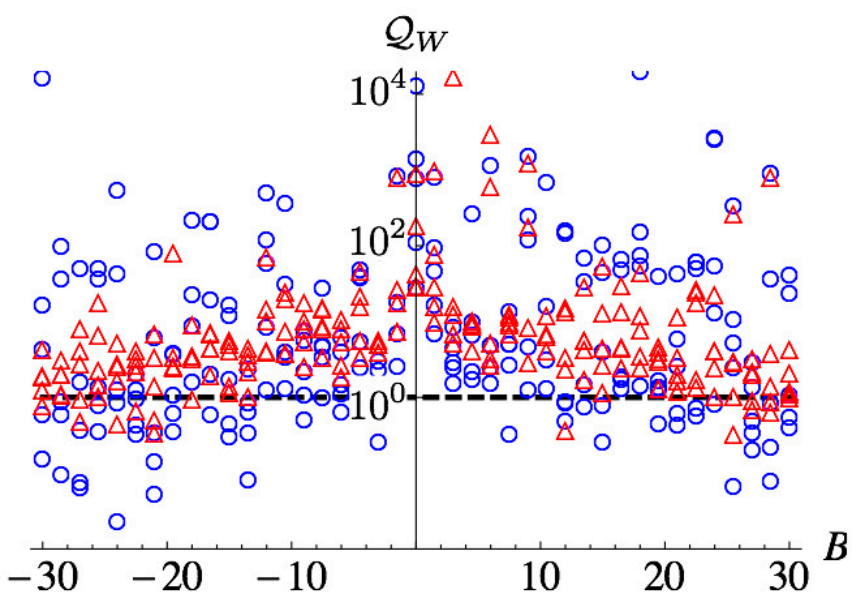

FIG. 4. Scatter plots of the uncertainty products for work done by $\boldsymbol{f}_{12}$ (blue circle) and $\boldsymbol{f}_{23}$ (red triangle) in threedimensional motion with a constant magnetic field $\boldsymbol{B}=$ $(0,0, B)$. Each data point is determined by a parameter set $\left(\kappa_{12}, \kappa_{23}, \kappa_{31}, m, B\right)$ that satisfies the stability condition. The parameters $\kappa_{12}, \kappa_{23}, \kappa_{31} \in[0,10]$ and $m \in[0,1]$ are randomly chosen, while $B \in[-30,30]$ is chosen at a constant interval of 1.5. The other parameters are fixed as $\gamma=k=T=1$.

the winding number current for two different weighting factors $\phi(\boldsymbol{z})=1$ and $\phi(\boldsymbol{z})=x_{1}$. A conceptually similar current as the former one was recently used in 32 in the context of biochemical oscillations. The uncertainty products for both weights are depicted in Fig. 3 for random parameters. The results show that the uncertainty product goes well below 1 in the latter case. In the former case, however, it seems to be bounded from below by 1 in the margin of error. In this special case the contribution to the current is independent from the radial distance and thus the localization of motion does not affect the current as strongly.

Next we consider a three-dimensional variant of the model. The motion of the particle is then described by the position $\boldsymbol{x}=\left(x_{1}, x_{2}, x_{3}\right)^{\mathrm{T}}$ and the velocity $\boldsymbol{v}=\left(v_{1}, v_{2}, v_{3}\right)^{\mathrm{T}}$. Three mutually perpendicular external torques are applied to the particle. They are given by $\boldsymbol{f}_{12}=\kappa_{12}\left(x_{2},-x_{1}, 0\right)^{\mathrm{T}}, \boldsymbol{f}_{23}=\kappa_{23}\left(0, x_{3},-x_{2}\right)^{\mathrm{T}}$, and $\boldsymbol{f}_{31}=\kappa_{31}\left(x_{3}, 0,-x_{1}\right)^{\mathrm{T}}$, respectively. The strengths of torques $\kappa_{12}, \kappa_{23}$, and $\kappa_{31}$ are in general different from each other. Fig. 4 shows numerically calculated uncertainty products of the work done by the torques $\boldsymbol{f}_{12}$ and $\boldsymbol{f}_{23}$ in the presence of a constant magnetic field $\boldsymbol{B}=(0,0, B)$. In the absence of the magnetic field, no violation of the thermodynamic uncertainty relation is observed in both cases. When the magnetic field is turned on, the uncertainty product for the work done by either torque goes below the usual bound of 1 . This is due to the localization of the projected motion onto the $x_{1} x_{2}$ plane and $x_{2} x_{3}$-plane. However, the localization effect on the $x_{1} x_{2}$-plane is stronger. As a consequence, the uncertainty product of the work done by $\boldsymbol{f}_{12}$ decreases more rapidly compared to the work done by $\boldsymbol{f}_{23}$. These nu- merical case studies suggest that the lower bound on the uncertainty product depends on the current of interest.

\section{CONCLUDING PERSPECTIVE}

We have investigated the effect of a magnetic field on the thermodynamic uncertainty relation through a driven charged Brownian particle in a magnetic field. We have focused on the work current done by an external torque. The physical mechanism of the violation of the thermodynamic uncertainty relation due to a magnetic field arises, since, on the one hand, the motion of the particle becomes localized due to the magnetic Lorentz force, which reduces dissipation. On the other hand, the uncertainty of the work current does not increase with increasing strength of the magnetic field. Thus the usual trade-off between dissipation and uncertainty of the work current does not apply in the presence of a magnetic field, resulting in a violation of the thermodynamic uncertainty relation. We also found that the lower bound on the uncertainty product depends on the current of interest as indicated by numerical results for winding number currents in two-dimensions and for work currents in the three-dimensional analogue of the harmonically trapped, driven particle.

This study leaves at least two general questions for future research on currents in underdamped dynamics in a magnetic field. First, can we classify the conditions under which the thermodynamic uncertainty relation still holds? In our two-dimensional system, the relative sign between magnetic field and torque has been crucial. How, if at all, can this finding be generalized? Second, for the putative other class, does there exist a universal weaker bound that is as transparent as the thermodynamic uncertainty relation? Finally, understanding the mechanism behind the violation of the thermodynamic uncertainty relation in the presence of a magnetic field might help to understand its validity for underdamped motion without a magnetic field.

\section{ACKNOWLEDGEMENTS}

H.-M. C. is supported by the the National Research Foundation of Korea (Grant No. 2018R1A6A3A03010776).

\section{Appendix A: Steady state distributions}

A linear stability analysis provides the condition that the probability distribution of $\boldsymbol{z}$ eventually converges to a steady state distribution. This happens only when all the real parts of the eigenvalues of the matrix $A$ are positive. The eigenvalues are given by the solutions of the two 
quadratic equations of $\psi$

$$
\begin{aligned}
& m \psi^{2}-(\gamma+i B) \psi+(k+i \kappa)=0, \\
& m \psi^{2}-(\gamma-i B) \psi+(k-i \kappa)=0 .
\end{aligned}
$$

By solving the equations, we get the four eigenvalues as

$$
\psi_{1}=\frac{\gamma+i B+\sqrt{\Psi}}{2 m}, \quad \psi_{2}=\frac{\gamma+i B-\sqrt{\Psi}}{2 m},
$$

and the complex conjugates $\psi_{1}^{*}$ and $\psi_{2}^{*}$, where $\Psi=(\gamma+$ $i B)^{2}-4 m(k+i \kappa)$. The real parts of the eigenvalues take on the two values

$$
\frac{1}{2 m}\left(\gamma \pm \sqrt{\frac{|\Psi|+\operatorname{Re}\{\Psi\}}{2}}\right)
$$

which should both be positive. Consequently, the system is stable only when the condition

$$
\gamma k+\kappa B-\kappa^{2} m / \gamma=\gamma K>0
$$

holds 19].

The steady state distribution of the multivariate Ornstein-Uhlenbeck process is a multivariate Gaussian with zero mean. The covariance matrix $C=\left\langle\boldsymbol{z} \boldsymbol{z}^{\mathrm{T}}\right\rangle_{\mathrm{ss}}$ is given by solving the matrix equation $A C+C A^{T}=2 \mathrm{D}[25]$. In the end we get the steady state distribution as

$$
P_{\mathrm{sS}}(\boldsymbol{z})=\frac{1}{\sqrt{\operatorname{det}(2 \pi \mathrm{C})}} \exp \left(-\frac{1}{2} \boldsymbol{z} \cdot \mathrm{C}^{-1} \boldsymbol{z}\right),
$$

where

$$
\mathrm{C}=\frac{T}{\gamma K}\left(\begin{array}{cccc}
\gamma & 0 & 0 & -\kappa \\
0 & \gamma & \kappa & 0 \\
0 & \kappa & (\gamma k+\kappa B) / m & 0 \\
-\kappa & 0 & 0 & (\gamma k+\kappa B) / m
\end{array}\right)
$$

In polar coordinates, the position of the particle is identified by the radial distance from the origin $r=|\boldsymbol{x}|$ and the polar angle $\theta=\tan ^{-1}\left(x_{2} / x_{1}\right)$. By changing variables and then marginalizing them, we get the probability distribution of the radial distance $r$ as

$$
P_{\mathrm{ss}}(r)=\frac{K r}{T} \exp \left(-\frac{K r^{2}}{2 T}\right)
$$

and the conditional distribution of the angular velocity $\dot{\theta}$ for a given $r$ as

$$
P_{\mathrm{ss}}(\dot{\theta} \mid r)=\sqrt{\frac{m r^{2}}{2 \pi T}} \exp \left[-\frac{m r^{2}}{2 T}\left(\dot{\theta}+\frac{\kappa}{\gamma}\right)^{2}\right] .
$$

From $\mathrm{A} 7$ and $\mathrm{A} 8$, we arrive at

$$
\langle\dot{W}\rangle_{\mathrm{ss}}=-\kappa\left\langle r^{2} \dot{\theta}\right\rangle_{\mathrm{ss}}=\frac{\kappa^{2}}{\gamma}\left\langle r^{2}\right\rangle_{\mathrm{ss}}=\frac{2 \kappa^{2} T}{\gamma K}
$$

with $K$ as defined in equation A4.

\section{Appendix B: Exact expressions of mean value and diffusion coefficient}

The exact mean value and diffusion coefficient of the work $W(t)$ can be obtained from the scaled cumulant generating function

$$
\lambda(h)=\lim _{t \rightarrow \infty} \frac{1}{t} \ln \left\langle e^{h W(t)}\right\rangle_{\mathrm{ss}} .
$$

For brevity, we rewrite the rate of work as $\dot{W}(t)=\dot{z}(t) \circ$ $\mathrm{W} \boldsymbol{z}(t)$ with

$$
\mathrm{W}=\left(\begin{array}{cccc}
0 & \kappa & 0 & 0 \\
-\kappa & 0 & 0 & 0 \\
0 & 0 & 0 & 0 \\
0 & 0 & 0 & 0
\end{array}\right)
$$

The scaled cumulant generating function is equivalent to the largest eigenvalue of the tilted Fokker-Planck operator [29]

$$
\mathcal{L}^{\dagger}=-\boldsymbol{z} \cdot \mathrm{A}^{\mathrm{T}}\left(\boldsymbol{\nabla}_{\boldsymbol{z}}+h \mathrm{~W} \boldsymbol{z}\right)+\left(\boldsymbol{\nabla}_{\boldsymbol{z}}+h \mathrm{~W} \boldsymbol{z}\right) \cdot \mathrm{D}\left(\boldsymbol{\nabla}_{\boldsymbol{z}}+h \mathrm{~W} \boldsymbol{z}\right) .
$$

We use the Gaussian ansatz

$$
g(\boldsymbol{z}, h)=\exp \left(-\frac{1}{2} \boldsymbol{z} \cdot \mathrm{G}(h) \boldsymbol{z}\right)
$$

for the left eigenfunction of the Fokker-Planck operator corresponding to the largest eigenvalue $\lambda(h)$ with a symmetric matrix $\mathrm{G}(h)$ 22. Since the untilted FokkerPlanck operator preserves probability, the constant function is the left eigenfunction with largest eigenvalue for $z=0$ and thus $\mathrm{G}(0)=0$. From the eigenvalue equation $\mathcal{L}^{\dagger} g(\boldsymbol{z}, h)=\lambda(h) g(\boldsymbol{z}, h)$, the scaled cumulant generating function follows as

$$
\lambda(h)=\operatorname{tr}\{\mathrm{D}[h \mathrm{~W}-\mathrm{G}(h)]\} .
$$

The matrix $\mathrm{G}(h)$ is determined by the identity

$$
\mathrm{A}^{\mathrm{T}}(h \mathrm{~W}-\mathrm{G})+(h \mathrm{~W}-\mathrm{G})^{\mathrm{T}} \mathrm{A}=2(h \mathrm{~W}-\mathrm{G})^{\mathrm{T}} \mathrm{D}(h \mathrm{~W}-\mathrm{G}) .
$$

It is hard to determine the matrix $\mathrm{G}(h)$ by solving the nonlinear equation in (B6). Nevertheless, the cumulants can be obtained from a series expansion of $\lambda(h)$ at $h=0$. Provided that the matrix $\mathrm{G}(h)$ is expanded as a series $\mathrm{G}(h)=\sum_{n=1}^{\infty} \mathrm{G}_{n} h^{n}$, the mean currents and diffusion coefficients are given by

$$
\langle\dot{W}\rangle_{\mathrm{ss}}=\left.\frac{\partial \lambda}{\partial h}\right|_{h=0}=\operatorname{tr}\left\{\mathrm{D}\left(\mathrm{W}-\mathrm{G}_{1}\right)\right\}
$$

and

$$
D_{W}=\left.\frac{1}{2} \frac{\partial^{2} \lambda}{\partial h^{2}}\right|_{h=0}=-\operatorname{tr}\left\{\mathrm{DG}_{2}\right\},
$$

respectively. The matrices $\mathrm{G}_{n}$ are determined by solving B6 order by order in powers of $h$. The first two orders give the identities

$$
\begin{aligned}
A^{\mathrm{T}}\left(\mathrm{W}-\mathrm{G}_{1}\right)+\left(\mathrm{W}-\mathrm{G}_{1}\right)^{\mathrm{T}} \mathrm{A} & =0, \\
\mathrm{~A}^{\mathrm{T}} \mathrm{G}_{2}+\mathrm{G}_{2} \mathrm{~A}+2\left(\mathrm{~W}-\mathrm{G}_{1}\right)^{\mathrm{T}} \mathrm{D}\left(\mathrm{W}-\mathrm{G}_{1}\right) & =0 .
\end{aligned}
$$


From these identities and the relation $A C+C A^{T}=2 D$, we get

$$
\langle\dot{W}\rangle_{\mathrm{ss}}=\operatorname{tr}\left\{\mathrm{ACW}_{a}\right\}
$$

and

$$
D_{W}=\operatorname{tr}\left\{\mathrm{ACW}_{a} \mathrm{C}\left(\mathrm{W}^{\mathrm{T}}-\mathrm{G}_{1}\right)\right\},
$$

where $\mathrm{W}_{a}=\left(\mathrm{W}-\mathrm{W}^{\mathrm{T}}\right) / 2$ is the asymmetric part of the matrix $W$. Thus we get the exact expressions of the mean current and the diffusion coefficient of the work current by finding $G_{1}$, which are given in $(9)$ and $(10)$ in terms of the specified dimensionless parameters.

Analogously the heat current can also be rewritten as $\dot{Q}(t)=\dot{\boldsymbol{z}}(t) \circ \mathrm{Q} \boldsymbol{z}(t)$ with

$$
\mathrm{Q}=\left(\begin{array}{cccc}
-k & \kappa & 0 & B \\
-\kappa & -k & -B & 0 \\
0 & 0 & -m & 0 \\
0 & 0 & 0 & -m
\end{array}\right)
$$

The mean value and diffusion coefficient of the heat current are given by a similar procedure in which the matrix W is replaced with $\mathrm{Q}$. The mean value and diffusion coefficient of the heat current are same with those of the work current. Consequently, the uncertainty product of the heat current is same as that of the work current.

\section{Appendix C: Scaling behavior of diffusion coefficient of work current}

In the following, we analyze the scaling behavior of the diffusion coefficient of the work current. We do this by rewriting the diffusion coefficient (6) as a Green-Kubo expression

$$
D_{W}=\lim _{t \rightarrow \infty} \int_{0}^{t} d s\langle\delta \dot{W}(s) \delta \dot{W}(t)\rangle_{\mathrm{ss}},
$$

where $\delta \dot{W}(t)=\dot{W}(t)-\langle\dot{W}(t)\rangle$. The correlation function of the work current $\langle\delta \dot{W}(s) \delta \dot{W}(t)\rangle_{\text {ss }}$ can be expressed as a sum of four-point correlation functions of $\boldsymbol{z}(t)$. Since the steady state distribution and transition probability of $\boldsymbol{z}(t)$ are Gaussian, the four-point correlation functions can be divided into two-point correlation functions using Wick's theorem. Thus we have

$$
\begin{aligned}
\langle\delta \dot{W}(s) \delta \dot{W}(t)\rangle_{\mathrm{ss}}=2 \kappa^{2} & \left\langle\left\langle x_{1}(s) x_{1}(t)\right\rangle_{\mathrm{ss}}\left\langle v_{1}(s) v_{1}(t)\right\rangle_{\mathrm{ss}}\right. \\
& +\left\langle x_{1}(s) x_{2}(t)\right\rangle_{\mathrm{ss}}\left\langle v_{1}(s) v_{2}(t)\right\rangle_{\mathrm{ss}} \\
& -\left\langle x_{1}(s) v_{2}(t)\right\rangle_{\mathrm{ss}}\left\langle v_{1}(s) x_{2}(t)\right\rangle_{\mathrm{ss}} \\
& \left.-\left\langle x_{1}(s) v_{1}(t)\right\rangle_{\mathrm{ss}}\left\langle v_{1}(s) x_{1}(t)\right\rangle_{\mathrm{ss}}\right)
\end{aligned}
$$

where the relations $\left\langle x_{1}(s) x_{1}(t)\right\rangle_{\mathrm{ss}}=\left\langle x_{2}(s) x_{2}(t)\right\rangle_{\mathrm{ss}}$, $\left\langle x_{2}(s) x_{1}(t)\right\rangle_{\mathrm{ss}}=-\left\langle x_{1}(s) x_{2}(t)\right\rangle_{\mathrm{ss}}$, and their time derivatives are used. These relations come from the fact that the physics of the system is not affected by the relabelling coordinates $\left(x_{1}, x_{2}\right) \rightarrow\left(x_{2},-x_{1}\right)$ due to the spatial isotropy of the system. Additionally, an integration by parts leads to

$$
\begin{array}{rl}
D_{W}=4 \kappa^{2} \lim _{t \rightarrow \infty} \int_{0}^{t} & d s\left(\left\langle x_{1}(s) x_{1}(t)\right\rangle_{\mathrm{ss}}\left\langle v_{1}(s) v_{1}(t)\right\rangle_{\mathrm{ss}}\right. \\
& \left.+\left\langle x_{1}(s) x_{2}(t)\right\rangle_{\mathrm{ss}}\left\langle v_{1}(s) v_{2}(t)\right\rangle_{\mathrm{ss}}\right) .
\end{array}
$$

The two-point correlation functions of $\boldsymbol{z}(t)$ involving two different times $s$ and $t$ are determined by the matrices $\mathrm{A}$ and $\mathrm{C}$ as $\left\langle\boldsymbol{z}(t-\tau) \boldsymbol{z}^{\mathrm{T}}(t)\right\rangle_{\mathrm{ss}}=\left\langle\boldsymbol{z}(t) \boldsymbol{z}^{\mathrm{T}}(t+\tau)\right\rangle_{\mathrm{ss}}=$ $\mathrm{C} e^{-\mathrm{A}^{\mathrm{T}} \tau}$. The matrix exponential can be decomposed as

$$
e^{-\mathrm{A}^{\mathrm{T}} \tau}=\sum_{j=1}^{2}\left(e^{\psi_{j} \tau} \boldsymbol{l}_{j} \boldsymbol{r}_{j}^{\mathrm{T}}+e^{\psi_{j}^{*} \tau} \boldsymbol{l}_{j}^{*}\left(\boldsymbol{r}_{j}^{*}\right)^{\mathrm{T}}\right)
$$

where $\boldsymbol{l}_{j}$ and $\boldsymbol{r}_{j}$ are the left and right eigenvector corresponding to the eigenvalue $\psi_{j}$, respectively. The eigenvectors are orthonormal, namely, $\boldsymbol{l}_{j} \cdot \boldsymbol{r}_{k}=\delta_{j k}$ and $\boldsymbol{l}_{j} \cdot \boldsymbol{r}_{k}^{*}=0$. Up to normalization, they are given by

$$
\begin{aligned}
\boldsymbol{l}_{1} & \propto\left(\psi_{2}, i \psi_{2}, 1, i\right)^{\mathrm{T}}, \\
\boldsymbol{l}_{2} & \propto\left(\psi_{1}, i \psi_{1}, 1, i\right)^{\mathrm{T}}, \\
\boldsymbol{r}_{1} & \propto\left(1,-i,-\psi_{1}, i \psi_{1}\right)^{\mathrm{T}}, \\
\boldsymbol{r}_{2} & \propto\left(1,-i,-\psi_{2}, i \psi_{2}\right)^{\mathrm{T}} .
\end{aligned}
$$

Apart from those eigenvectors the correlation function has time dependent contributions with different relaxation times and oscillation frequencies, which are determined by the eigenvalues $\psi_{1}$ and $\psi_{2}$. Thus the time dependence of $\langle\delta \dot{W}(t-\tau) \delta \dot{W}(t)\rangle_{\mathrm{ss}}$ is determined by $e^{-\left(\psi_{j}+\psi_{k}\right) \tau}$ and $e^{-\left(\psi_{j}+\psi_{k}^{*}\right) \tau}$ for all pairs of $(j, k)$ with some prefactors.

In the strong magnetic field limit, the eigenvalues of $A$ up to the leading order are given by $\psi_{1}=(\gamma+i B) / m$, $\psi_{2}=(\kappa-i k) / B$ and their complex conjugates. The twopoint correlation function of the position and that of the velocity up to the order of $B^{-2}$ are given by

$$
\left\langle\boldsymbol{x}(t-\tau) \boldsymbol{x}^{\mathrm{T}}(t)\right\rangle_{\mathrm{ss}} \simeq \frac{m T}{B^{2}} e^{-\gamma \tau / m} \mathrm{R}(B \tau / m)+\frac{\gamma T}{\kappa B}\left(1+\frac{\gamma k}{\kappa B}\right) e^{-\kappa \tau / B} \mathrm{R}(k \tau / B),
$$




$$
\left\langle\boldsymbol{v}(t-\tau) \boldsymbol{v}^{\mathrm{T}}(t)\right\rangle_{\mathrm{ss}} \simeq T\left(\frac{1}{m}+\frac{\gamma k+\kappa B-\kappa^{2} m / \gamma}{\gamma B^{2}}\right) e^{-\gamma \tau / m} \mathrm{R}(B \tau / m)
$$

with

$$
\mathrm{R}(\varphi)=\left(\begin{array}{cc}
\cos \varphi & -\sin \varphi \\
\sin \varphi & \cos \varphi
\end{array}\right)
$$

The leading terms of the cross correlation functions $\left\langle\boldsymbol{x}(t-\tau) \boldsymbol{v}^{\mathrm{T}}(t)\right\rangle_{\mathrm{ss}}$ and $\left\langle\boldsymbol{v}(t-\tau) \boldsymbol{x}^{\mathrm{T}}(t)\right\rangle_{\mathrm{ss}}$ are of the order $B^{-1}$. Thus the correlation function of the work current in $\mathrm{C} 2$ is primarily determined by

$$
\begin{aligned}
\left\langle x_{1}(t-\tau)\right. & \left.x_{1}(t)\right\rangle_{\mathrm{ss}}\left\langle v_{1}(t-\tau) v_{1}(t)\right\rangle_{\mathrm{ss}} \\
& \simeq\left(\gamma T^{2} /(\kappa m B)\right) e^{-\gamma \tau / m} \cos (B \tau / m)
\end{aligned}
$$

which like the velocity and position correlation functions follows oscillations that are decaying over time. The integration of this leading term over $\tau$ gives a result of the order $B^{-3}$. This is, however, a subleading term of the diffusion coefficient.

The leading term of the diffusion coefficient arises from the integration of subleading, additional terms that correspond to $e^{-\left(\psi_{1}+\psi_{1}^{*}\right) \tau}$. The product of the first term of (C6) and the leading term of C7 gives

$$
\begin{aligned}
I_{1} & =\int_{0}^{\infty} d \tau\left\langle x_{1}(t-\tau) x_{1}(t)\right\rangle_{\mathrm{ss}}\left\langle v_{1}(t-\tau) v_{1}(t)\right\rangle_{\mathrm{ss}} \\
& =\frac{T^{2}}{B^{2}} \int_{0}^{\infty} d \tau e^{-2 \gamma \tau / m} \cos ^{2}\left(\frac{B \tau}{m}\right)+\mathcal{O}\left(B^{-3}\right),
\end{aligned}
$$

$$
\begin{aligned}
I_{2} & =\int_{0}^{\infty} d \tau\left\langle x_{1}(t-\tau) x_{2}(t)\right\rangle_{\mathrm{ss}}\left\langle v_{1}(t-\tau) v_{2}(t)\right\rangle_{\mathrm{ss}} \\
& =\frac{T^{2}}{B^{2}} \int_{0}^{\infty} d \tau e^{-2 \gamma \tau / m} \sin ^{2}\left(\frac{B \tau}{m}\right)+\mathcal{O}\left(B^{-3}\right),
\end{aligned}
$$

and finally

$$
D_{W}=4 \kappa^{2}\left(I_{1}+I_{2}\right)=\frac{2 \kappa^{2} m T^{2}}{\gamma B^{2}}+\mathcal{O}\left(B^{-3}\right) .
$$

The oscillations do not cancel in the integrations C10 and C11 since the integrands are positive. The mean work current scales as $\langle\dot{W}\rangle_{\mathrm{ss}} \sim B^{-1}$ for large $B$ provided that $\kappa B>0$. Thus the squared relative uncertainty $\epsilon_{W}^{2}=2 D_{W} /\langle\dot{W}\rangle_{\text {ss }}^{2}$ converges to a constant as $B$ increases. In the small mass limit $m B \ll 1$, the leading order of the diffusion coefficient becomes $B^{-3}$, which results in a decreasing uncertainty $\epsilon_{W}^{2} \sim B^{-1}$.
[1] A. C. Barato and U. Seifert, Phys. Rev. Lett. 114, 158101 (2015).

[2] T. R. Gingrich, J. M. Horowitz, N. Perunov, and J. L. England, Phys. Rev. Lett. 116, 120601 (2016).

[3] U. Seifert, Physica A 504, 176 (2018).

[4] K. Proesmans and C. Van den Broeck, Europhys. Lett. 119, 20001 (2017).

[5] A. Dechant, J. Phys. A: Math. Theor. 52, 035001 (2018).

[6] M. Polettini, A. Lazarescu, and M. Esposito, Phys. Rev. E 94, 052104 (2016).

[7] T. R. Gingrich, G. M. Rotskoff, and J. M. Horowitz, J. Phys. A 50, 184004 (2017).

[8] A. Dechant and S.-I. Sasa, J. Stat. Mech.: Theor. Exp. 2018, 063209 (2018).

[9] A. Dechant and S.-I. Sasa (2018), arXiv:1804.08250v2 [cond-mat.stat-mech].

[10] P. Pietzonka, F. Ritort, and U. Seifert, Phys. Rev. E 96, 012101 (2017).

[11] J. M. Horowitz and T. R. Gingrich, Phys. Rev. E 96, 020103(R) (2017).

[12] S. Pigolotti, I. Neri, E. Roldán, and F. Jülicher, Phys. Rev. Lett. 119, 140604 (2017).

[13] A. C. Barato, R. Chetrite, A. Faggionato, and D. Gabrielli, New. J. Phys. 20, 103023 (2018).

[14] T. Koyuk, U. Seifert, and P. Pietzonka, J. Phys. A: Math. Theor. 52, 02LT02 (2019).
[15] L. P. Fischer, P. Pietzonka, and U. Seifert, Phys. Rev. E 97, 022143 (2018).

[16] K. Brandner, T. Hanazato, and K. Saito, Phys. Rev. Lett. 120, 090601 (2018).

[17] K. Macieszczak, K. Brandner, and J. P. Garrahan, Phys. Rev. Lett. 121, 130601 (2018).

[18] T. Van Vu and Y. Hasegawa (2019), arXiv:1901.05715v1 [cond-mat.stat-mech].

[19] S. Lee and C. Kwon (2019), arXiv:1901.02622v1 [condmat.stat-mech].

[20] R. Filliger and P. Reimann, Phys. Rev. Lett. 99, 230602 (2007).

[21] C. Kwon, J. D. Noh, and H. Park, Phys. Rev. E 83 , 061145 (2011).

[22] P. Pietzonka and U. Seifert, Phys. Rev. Lett. 120, 190602 (2018).

[23] K. H. Chiang, C. L. Lee, P. Y. Lai, and Y. F. Chen, Phys. Rev. E 96, 032123 (2017).

[24] A. Argun, J. Soni, L. Dabelow, S. Bo, G. Pesce, R. Eichhorn, and G. Volpe, Phys. Rev. E 96, 052106 (2017).

[25] C. Gardiner, Stochastic Methods: A Handbook for the Natural and Social Sciences (Springer, New York, 2010), 4th ed.

[26] K. Sekimoto, Prog. Theor. Phys. Suppl. 130, 17 (1998).

[27] U. Seifert, Rep. Prog. Phys. 75, 126001 (2012).

[28] P. Pietzonka, A. C. Barato, and U. Seifert, Phys. Rev. E 
93, 052145 (2016).

[29] H. Touchette, Physica A 504, 5 (2018).

[30] Y. Hasegawa and T. Van Vu, arXiv (2019), arXiv:1902.06376v1 [cond-mat.stat-mech].
[31] K. Proesmans and J. Horowitz (2019), arXiv:1902.07008v2 [cond-mat.stat-mech].

[32] R. Marsland III, W. Cui, and J. Horowitz (2019), arXiv:1901.00548v2 [physics.bio-ph]. 